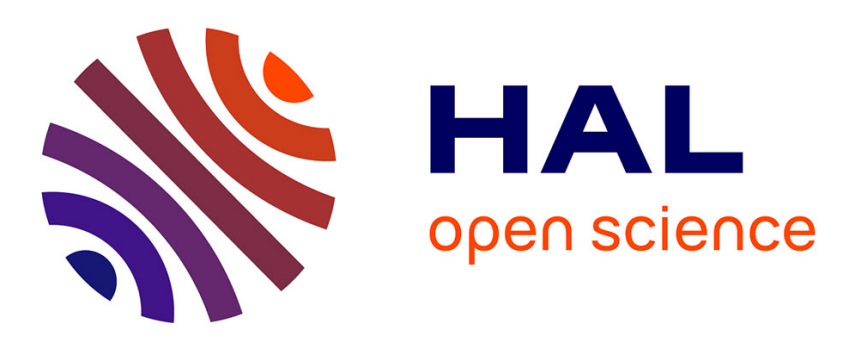

\title{
Automated visual grading of grain kernels by machine vision
}

Pierre Dubosclard, Stanislas Larnier, Hubert Konik, Ariane Herbulot, Michel

Devy

\section{- To cite this version:}

Pierre Dubosclard, Stanislas Larnier, Hubert Konik, Ariane Herbulot, Michel Devy. Automated visual grading of grain kernels by machine vision. 12th International Conference on Quality Control by Artificial Vision 2015, Jun 2015, Le Creusot, France. 10.1117/12.2182811 . hal-01355086

\section{HAL Id: hal-01355086 https://hal.science/hal-01355086}

Submitted on 22 Aug 2016

HAL is a multi-disciplinary open access archive for the deposit and dissemination of scientific research documents, whether they are published or not. The documents may come from teaching and research institutions in France or abroad, or from public or private research centers.
L'archive ouverte pluridisciplinaire HAL, est destinée au dépôt et à la diffusion de documents scientifiques de niveau recherche, publiés ou non, émanant des établissements d'enseignement et de recherche français ou étrangers, des laboratoires publics ou privés. 


\title{
Automated visual grading of grain kernels by machine vision
}

\author{
Pierre Dubosclard ${ }^{a, b}$, Stanislas Larnier ${ }^{a, b}$, Hubert Konik $^{c}$, \\ Ariane Herbulot ${ }^{a, b}$ and Michel Devy ${ }^{a, b}$ \\ ${ }^{a}$ CNRS, LAAS, 7 avenue du colonel Roche, F-31400 Toulouse, France; \\ ${ }^{b}$ Univ de Toulouse, UPS, LAAS, F-31400 Toulouse, France; \\ ${ }^{c}$ Laboratoire Hubert Curien, Saint-Etienne, France
}

\begin{abstract}
This paper presents two automatic methods for visual grading, designed to solve the industrial problem of evaluation of seed lots from the characterization of a representative sample. The sample is thrown in bulk onto a tray placed in a chamber for acquiring color image in a controlled and reproducible manner. Two image processing methods have been developed to separate, and then characterize each seed present in the image. A shape learning is performed on isolated seeds. Collected information is used for the segmentation. The first approach adopted for the segmentation step is based on simple criteria such as regions, edges and normals to the boundary. Marked point processes are used in the second approach, leading to tackle the problem by a technique of energy minimization. In both approaches, an active contour with shape prior is performed to improve the results. A classification is done on shape or color descriptors to evaluate the quality of the sample.
\end{abstract}

Keywords: image segmentation, visual grading, shape learning, marked point processes, active contours.

\section{INTRODUCTION}

This paper presents a global solution for an industrial problem: the visual grading of grain products. Millions of tons are exchanged each year on the cereal worldwide market, and with the increase of the demand for high quality grain, the need of an automatic system able to proceed this visual grading has become a major issue. The quality evaluation of grain is here addressed from the acquisition to the detection and the classification. The subject has been widely discussed during the last ten years. A number of studies have been conducted to classify grain based on physical attributes.

Some studies are applied on images with separated grain kernels. Kaur and Singh ${ }^{1}$ use support vector machine to classify and grade rice grain. From an image of isolated rice kernels and after the background threshold, some classic characteristics, such as for example area, length or perimeter, are extracted for each detected kernel. These features are then used by a trained support vector machine to grade and classify each rice kernel as broken, long or round. Augustin et al. ${ }^{2}$ presents a method based on neural network for the classification of rice kernels, regarding different shape and color features. Each kernel can be classify as complete, broken or color defect. These two methods of classification and grading provide good results on separated grain kernels, but can not be used to process touching seeds.

Other studies have been conducted on the grain segmentation. Yao. et al. ${ }^{3}$ and Faessel et al. ${ }^{4}$ focused on detection and separation of touching rice grain. They both work on a binary image obtained by a threshold to separate the objects from the background. Yao et al. ${ }^{3}$ then work on the contours and search the concave angles to connect them two at a time in order to detect objects boundaries. Faessel et al. ${ }^{4}$ used a mathematical morphology method on the binary image: a skeleton operation on the background. The open lines of the skeleton, without ending, are then combined under some constraints to obtain the objects boundaries. These two methods give good results on image of touching grain with low density of objects, but are not adapted for images with heaps and high density of seeds. Mebatsion et al. ${ }^{5}$ proposed a method to separate touching grain kernels, using an elliptic Fourier series approximation, based on boundary curvature values. This approach successfully separates most instances of touching kernels, but does not work with broken kernels or if the density is too important.

Finally, Zhang et al. ${ }^{6}$ proposed a segmentation and classification approach. An ellipse fitting algorithm separates the touching grain kernels. Morphological features are extracted for each kernel and a back-propagation 
neural network is employed for grain type classification. A comparison has been made between the features extracted from the algorithm and from images of physically separated kernels. The feature values obtained on both cases are quite similar, but the method is adapted for images with a few number of grain kernels.

The solution introduced in this paper addresses the learning, the segmentation and the classification topics for the visual grading of grain kernels. This is an automated solution that allows to handle different kind of kernel shapes: simple ones as ellipses and more complex ones as superellipses. ${ }^{7}$ These shapes are defined during a learning step. The segmentation process deals with touching grain kernels, with low or high densities, under the constraint of no seeds overlapping. Two segmentation approaches, deterministic and probabilistic, are presented and compared. They are extensions of previous works. ${ }^{8-10}$

\section{DATA ACQUISITION SYSTEM}

The acquisitions are performed in a dedicated cabin. ${ }^{8}$ It has been designed to provide stable condition, independently from external lighting. This cabin integrates a mono CMOS sensor color camera of 5 megapixels and a lighting solution. The camera equipped with a $5 \mathrm{~mm}$ lens has been placed at the distance of $400 \mathrm{~mm}$ from the object plan and offers a resolution on the object plan around 6 pixels per millimeter. The lighting system is composed of white LEDs. These LEDs have a continuous spectrum in the visible range and a good stability over time in term of luminous intensity. After the bulk loading of the grain seeds sample on a tray placed in the cabin, the image of the sample is saved. The seeds are spread in order to avoid overlapping.

\section{AUTOMATIC VISUAL GRADING}

The method is composed of three main steps: learning, segmentation and classification. For a given application, a learning has to be performed once before the other steps, to define the parameters of the segmentation.

\subsection{Learning}

The learning step is processed on a binarized image that distinguishes the objects from the background. This binarized image is obtained by using the Otsu method ${ }^{11}$ or by removing the background previously learned as the acquisition conditions are stable. The aims of the learning step are: 1) to evaluate the seed shapes of the considered sample, in order to provide to the segmentation algorithm the parameters that best characterize the objects; 2) to define some criteria that will be used for objects validation during the segmentation.

1) To evaluate a generic shape of the seeds, a mean shape is built from the isolated objects detected in the learning image. For a given number of level sets on the mean shape, we evaluate different parametric curves that best corresponds to a superellipse. ${ }^{7}$ The number of considered level sets defines the number of learned shapes that will be used during the segmentation step. The raw level sets can be used as learned shapes but the parametric curves show the advantage to provide a sharper contour.

2) In order to validate the objects detected during the segmentation step, some criteria are retained during the learning step. These criteria are chosen, depending of the application, between many common features (e.g. area, aspect ratio, roundness) and more advanced features (e.g. Hu moments, ${ }^{12}$ elliptic Fourier descriptors ${ }^{13}$ ).

\subsection{Segmentation}

Two different approaches have been developed to perform the detection of grain kernels in bulk. Both are using the same tools, but in a different manner; a comparison is made here. The deterministic approach considers only the supposed best result, whereas the probabilistic one introduces a stochastic aspect. Therefore the deterministic approach provides always the same results for the same image with the same learning parameters, while this statement is not true for the probabilistic approach. In term of computation time, the probabilistic approach is slower but can potentially avoid some false positive detections. In an industrial context, it is important to provide reproducible results, but it is obviously interesting to use a stochastic approach to prevent from wrong detections. Therefore a comparison is made here to determine the difference between both approaches in terms of reproducibility and accuracy.

These two methods are based on the same initial steps. The image is first binarized, according to the method used during the learning. In the next step, three indicators are defined to extract information from the input 

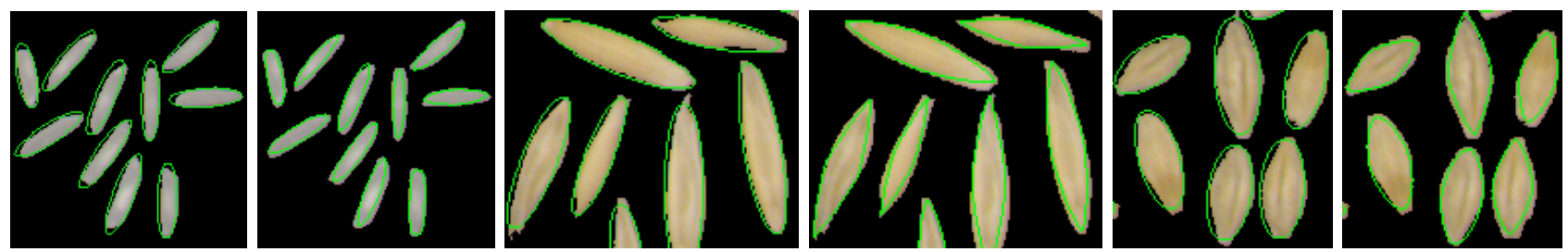

Figure 1. Fitting results on isolated grain kernels to illustrate the interest of superellipse (even) against ellipse (odd).

image. Based on a given learned shape, they provide: the matter ratio inside; the gradient gap on the boundary; the normal to the boundary. An energy map associates for each point of the input image a value based on these indicators. Different energy maps are obtained by considering different given shapes provided by the learning and different orientations. The number of considered orientations to compute these energy maps is a parameter of the segmentation step. The energy maps bring information on the placement of the objects in the image by providing for each pixel matching scores for the learned shapes at different orientations. It is more efficient with isolated seeds and on the boundary of seed heaps.

Referring to these energy maps, both segmentation approaches provide approximations of the grain kernels. These approximations are then improved to get the boundaries of the seeds by using an active contour with shape prior introduced by Bresson et al. ${ }^{14}$ This active contour returns an object which is compared to the criteria given by the learning to be validated. The energy maps are updated when an object is validated: the area of the object detected is then considered as part of the background, which makes the segmentation of heaps possible by tackling each heap progressively from its boundaries to its core.

\subsubsection{Deterministic approach}

The deterministic approach is an extension a previous work. ${ }^{9}$ It considers the best match provides by the energy maps. In other words, the shape that gives the best score regarding the energy maps is submitted to validation by the active contour with shape prior, and then compared with the learning criteria. If the object is validated, the energy maps are updated and the new best match is considered.

\subsubsection{Probabilistic approach}

The probabilistic approach is adapted from a previous method ${ }^{8}$ based on the marked point processes and birthand-death dynamic. A difference is that the shapes considered could be more complex than just ellipses. A second difference is that the maps computed during the initialization step $^{8}$ are replaced by the energy maps used in this paper. The matching scores of the energy maps are considered as probabilities. For each point of the image, an object is randomly created considering the probability of each oriented shape given by the energy maps. According to the birth-and-death dynamic with a detection step, ${ }^{8}$ the active contour is used to get the boundaries of the seed, and a comparison with some criteria of the learning data validate or invalidate the object.

\subsection{Classification}

The classification step is performed on the data provided by the segmentation process. For each detected seed, common features (e.g. area, perimeter, aspect ratio, roundness) and more advanced features (e.g. Hu moments, elliptic Fourier descriptors) are computed. A clustering method returns the size distribution of different classes of grain kernels, usually two, broken and intact. Quality control can also be made thanks to the color.

\section{EXPERIMENTS}

\subsection{Learning experiments}

Figure 1 illustrates the results of a fitting ellipse and a fitting superellipse algorithms on each isolated seed. One can note than with superellipse the grain kernels are better approximate. Simple curves were kept because human operators can more easily change the parameters if they want. In practice, instead to fit curves on isolated seeds, a mean map is extracted from isolated seeds. Then the fitting algorithms are performed on some level sets. 


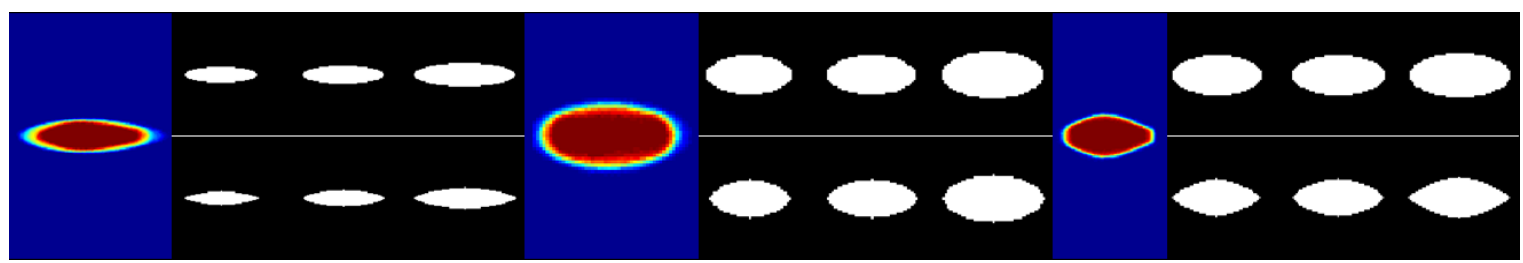

Figure 2. Three mean shapes of barley and wheat grain kernels with the associated ellipse and superellipse shapes.

Table 1. Percentage of detected objects and the mean error on the barycenters.

\begin{tabular}{|c|c|c|c|c|c|c|c|c|}
\cline { 6 - 9 } & \multicolumn{3}{|c|}{ Percentage of detected objects } & \multicolumn{4}{c|}{ Mean error on the barycenters } \\
\hline FE & FSE & FEAC & FSEAC & Species & FE & FSE & FEAC & FSEAC \\
\hline 97 & 100 & 100 & 100 & Barley 1 & 2.35 & 3.56 & 1.99 & 2.22 \\
\hline 97 & 99 & 98 & 98 & Barley 2 & 1.24 & 1.23 & 0.94 & 0.88 \\
\hline 95 & 97 & 98 & 100 & Rice & 2.13 & 3.38 & 1.28 & 1.16 \\
\hline 97 & 99 & 99 & 100 & Wheat & 1.30 & 1.28 & 0.91 & 0.79 \\
\hline
\end{tabular}

Figure 2 illustrates the mean map and some mean shapes extracted from the learning. There are wheat and two types of barley grain kernels. The first line presents three ellipse shapes and the second line, three superellipse ones. In this paper, the learning is performed on one hundred grain kernels.

\subsection{Evaluation criteria}

To evaluate the segmentation accuracy, a comparison is done with a "gold standard" segmentation image. This image is obtained by a human operator, who defines the boundaries of all the objects, present in the image. Some criteria are computed, considering the gold standard image as reference. The first step consists in matching the objects of both images, by considering the distance between the objects barycenters of the two images.

The following criteria are computed for each objects pair, with $\bar{\omega}$ and $\omega$ referring respectively to the object from the gold standard image and the one from the evaluated image. The first are classical errrors: the barycenter error: $e_{b}$; the orientation error: $e_{\theta}$; the area error: $e_{a}$; the perimeter error: $e_{p}$. Let $\operatorname{card}(\omega)$ be the cardinality of a set $\omega, b_{\omega}$ the barycenter of $\omega,\|$.$\| the Euclidean norm and \partial \omega$ the boundary of $\omega$. The errors formula are:

$$
e_{b}=\left\|b_{\bar{\omega}}-b_{\omega}\right\| ; e_{\theta}=\left|\theta_{\bar{\omega}}-\theta_{\omega}\right| ; e_{a}=|\operatorname{card}(\bar{\omega})-\operatorname{card}(\omega)| ; e_{p}=|\operatorname{card}(\partial \bar{\omega})-\operatorname{card}(\partial \omega)|
$$

Other known criteria are the false positive ratio: $F P$; the false negative ratio: $F N$; and the Dice similarity coefficient: ${ }^{15} D$. Let $\omega^{c}$ be the complement of $\omega$ and $d(.,$.$) the Euclidean distance. These other criteria are$ defined as follow:

$$
F P(\omega \mid \bar{\omega})=\operatorname{card}\left(\bar{\omega}^{c} \cap \omega\right) ; \quad F N(\omega \mid \bar{\omega})=\operatorname{card}\left(\bar{\omega} \cap \omega^{c}\right) ; \quad D(\bar{\omega}, \omega)=\frac{2 \operatorname{card}(\bar{\omega} \cap \omega)}{\operatorname{card}(\bar{\omega})+\operatorname{card}(\omega)}
$$

\subsection{Segmentation experiments}

The first experiments focus on a comparison with human operator and the deterministic approach in the case of high density touching grain kernels. Table 1 (left) provides the percentage of detected objects on experiments with touching grain kernels in high density. Four kinds of grain kernels: two barley species, rice and wheat are considered. Four deterministic approaches are compared. The first two are without the active contours with only the indicators to compare ellipse and superellipse fitting. The two others have in addition the second step with the active contours. The results show that the percentage of detect grain kernels is higher when the superellipse shapes are used. Most of the time, the active contours provides the same or improve the number of detected objects. Table 1 (right) provides also the mean error on the barycenters to demonstrate that the objects are detected and at the correct location.

This paragraph focuses on the interest of using superellipse instead of ellipse, both with active contours. For all the four studied grain kernels, for both methods, on average the orientation error is of 1 to 2 degrees. The perimeter and area errors are less than 10\%. Figure 3 presents a comparison of both methods with other 

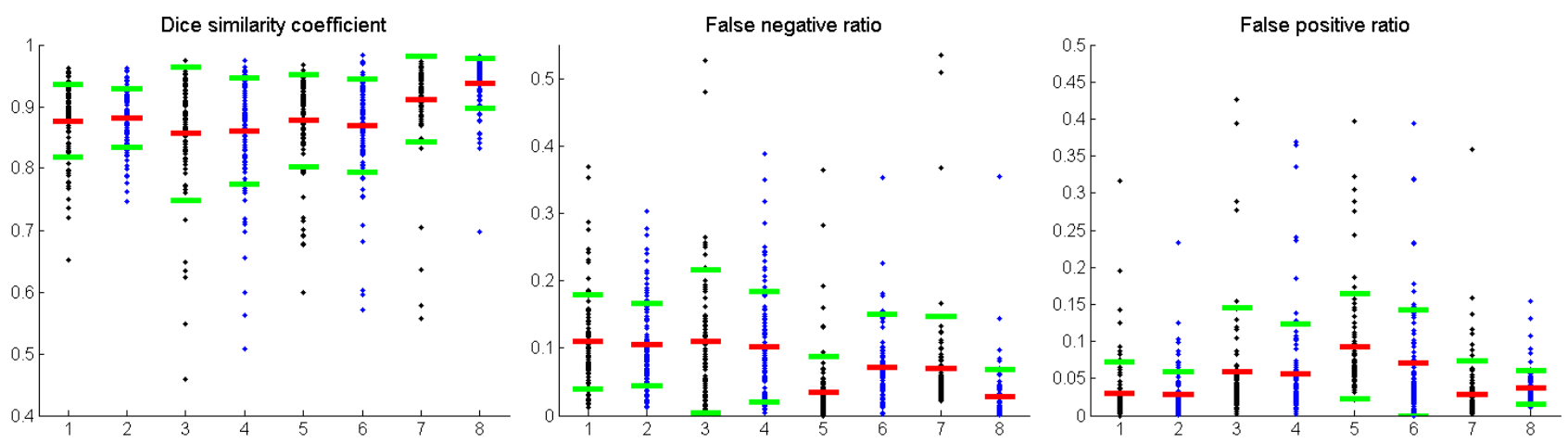

Figure 3. Diagram results on the deterministic method, barley 1 FEAC (1), barley 1 FSEAC (2), barley 2 FEAC (3), barley 2 FSEAC (4), rice FEAC (5), rice FSEAC (6), wheat FEAC (7), wheat FSEAC (8).
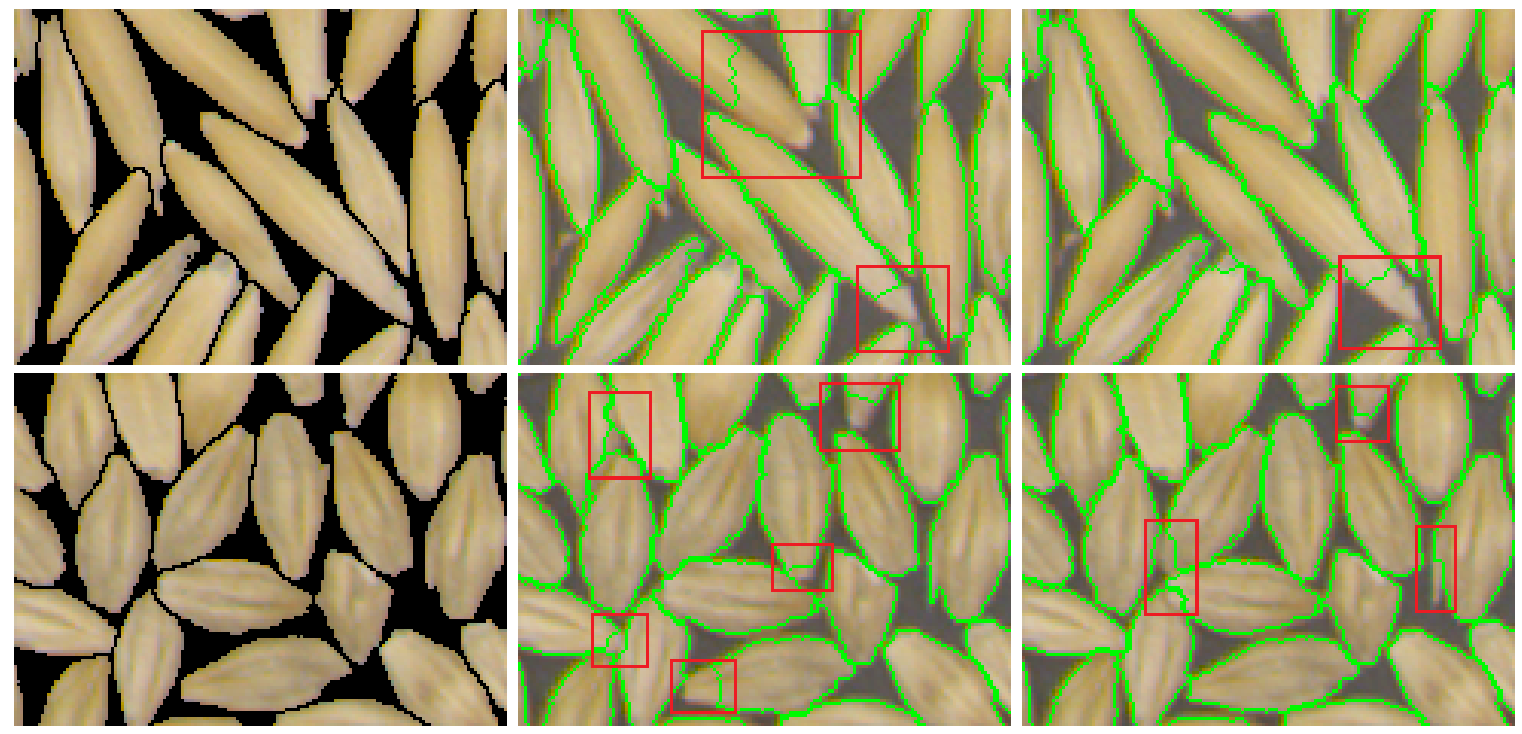

Figure 4. Segmentation results on touching barley grain kernels. From left to right, the human segmentation, the deterministic approach with ellipse shapes then with superllipse shapes. The red rectangles highligth segmentation problems.

evaluation criteria: Dice similarity coefficient, false negative ratio, false positive ratio. The red and green lines in each diagram represent respectively the mean and the mean plus or minus the standard deviation. Figure 4 presents a visual comparison on some of these segmentation results. Most of barley grain kernels of specie are well detected. There are some segmentation problems highlight with red rectangles. The use of the superellipse leads generally to better results compared to the ones with ellipse. Segmentation could be improved with shapes more accurate. For example, the second specie of barley is less symmetric than the other grain kernels.

Experiments have been conducted on the density level of the grain kernels, using the same sample, from isolated seeds to large heaps, in order to evaluate the effects of the density on the results of the segmentation approaches. Figure 5 provides shape statistics curves for the same rice sample with different density from isolated grain kernels to a large heap in the case of the deterministic approach. Two images are provided to present the extreme grain kernel dispositions. The purpose of this experiment is to be able to recognize the same sample. One can note than the major axis, perimeter and area statistics are quite similar. The minor axis is the one which present the more difference. This behavior can be explained by the fact that the pixel boundaries of a detected object cannot be at the same location of pixel boundaries of another object. This drawback has been also noted on comparison with human operators.

A comparison of the deterministic and probabilistic segmentation methods has also been done, to outline the benefits and the shortcomings of each approach. The main interest of the deterministic approach is its reproducibility. The probabilistic approach could or could not provide better results. On the studied experiments, 

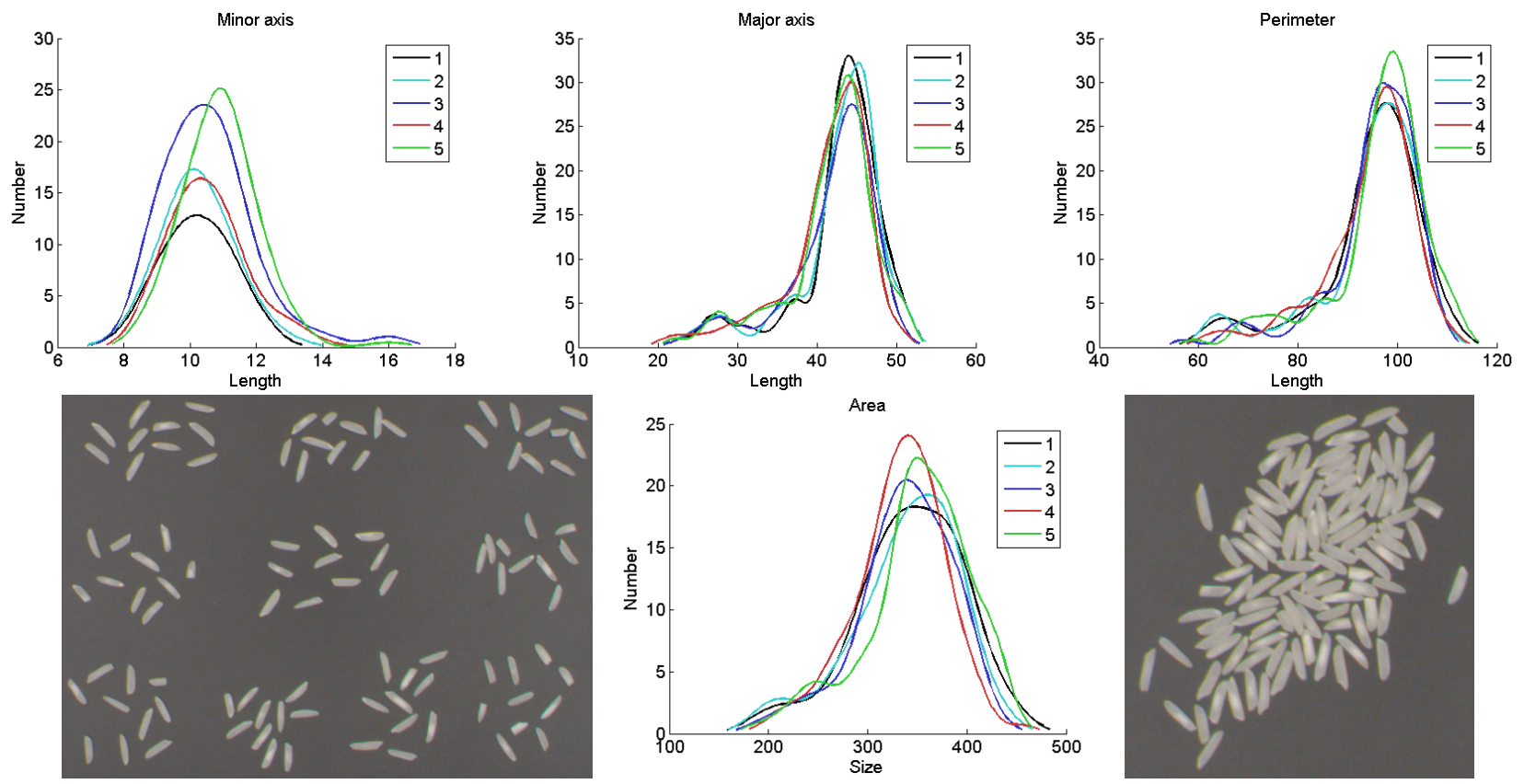

Figure 5. Comparison of shape statistics for the same rice sample with different density (1 isolated 5 heap).
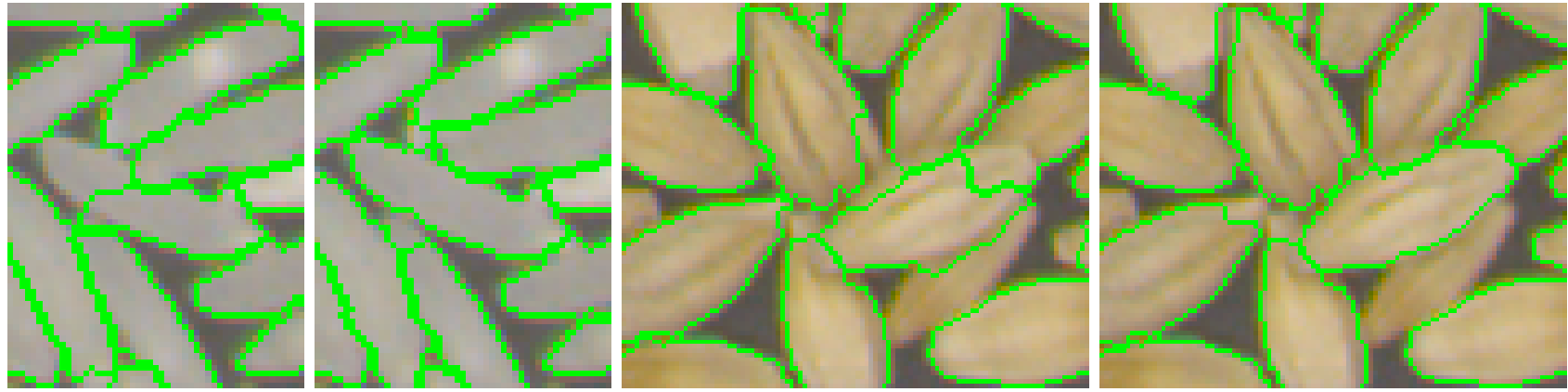

Figure 6. Segmentations of rice and barley grain kernels with deterministic method then probabilistic approach.

the evaluation criteria are very similar between both. Figure 6 illustrates some of the better results obtained with the probabilistic approach. Confidence rate on the segmentation could help to decide which of the deterministic or probabilistic methods provide the best result in a given area.

Figure 7 presents a visual grading experiment on the rice grain kernels in a large heap. The original image is given in Figure 5. In the same figure, thanks to the major axis, perimeter and area curves, one can note that there are intact and broken grain kernels. The left image in Figure 7 represents the curve on the area and a threshold value determined by the learning process. There are $20 \%$ of broken grain kernels. The right image in Figure 7 is a zoom on the segmentation results, the intact grain kernels have a blue cross on them, the broken ones have a red cross. On the example, there is a bad segmentation result which leads to a wrong classification of this grain kernel. It is one of the reasons why a confidence rate research is under way.

Figure 8 presents a color classification on pea grain kernels. The grain kernels are segmented with the deterministic approach. A mean is done on each color channels of each grain kernel. Some thresholds are applied to classify the grain kernel into three classes. The left image is the original image with a letter on each grain kernel. The right one is a zoom on some grain kernels, each line represent a class.

Confidence rates for the segmentation of each grain kernel are provided by the algorithm. It is an industrial demand. For visual grading, this confidence information could be taken into account in the quality prediction. Figure 9 illustrates the use of the energy map results as confidence rates. The grain kernels have crosses on 

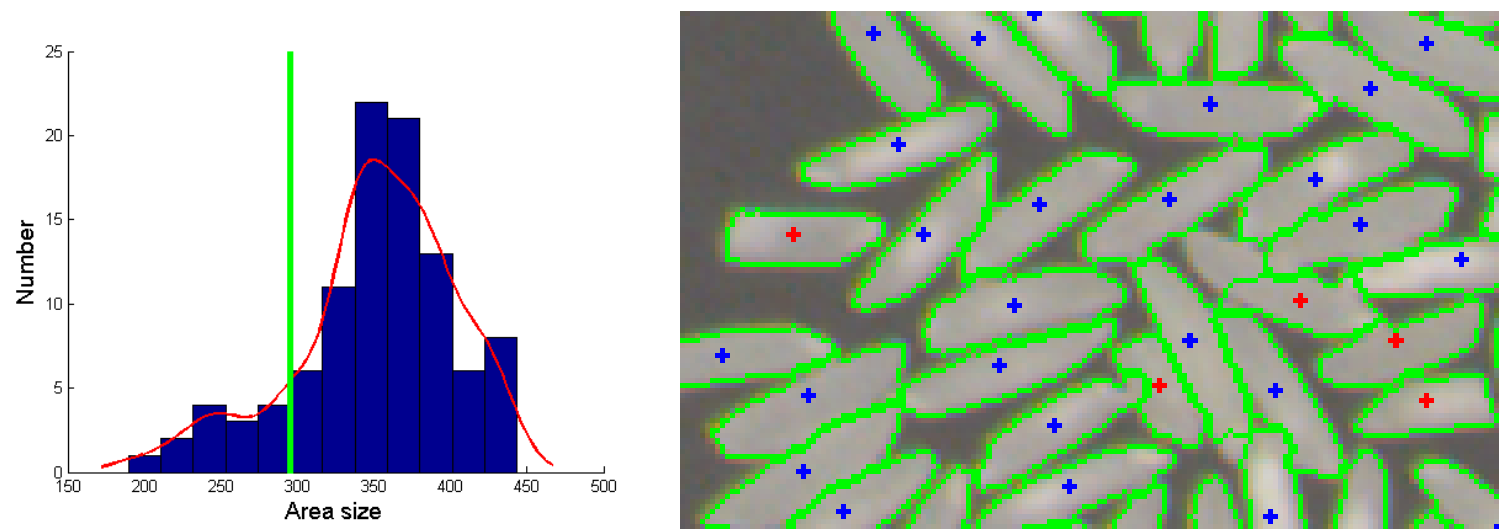

Figure 7. At left the area curve. At right, the segmentation with broken (red cross) and intact (blue cross) rice seeds.
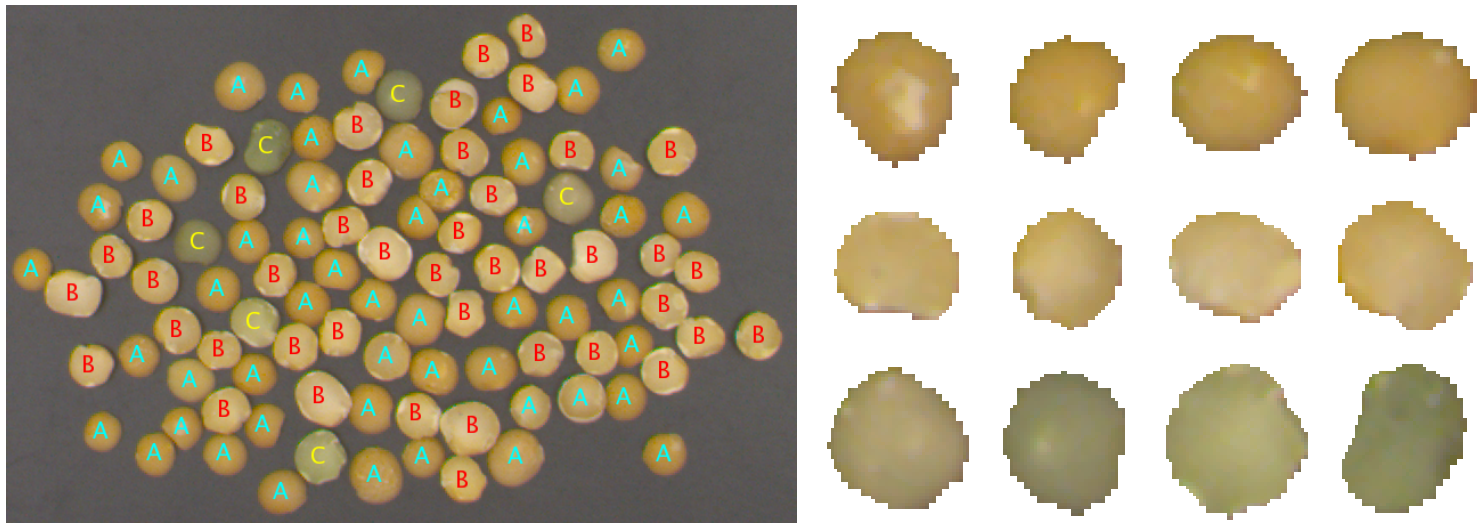

Figure 8. At left, the classified image of pea grain kernels. At right, some grain kernels, each line represents a class.

them. The blue, cyan and red crosses represent respectively the very good, good and bad confidences. One can note that the actual confidence rate reacts to segmentation problems due to overlapping grain kernels which is good. Unfortunately, sometimes, it also reacts on grain kernels which are too far away from the learning.

\section{CONCLUSION AND PROSPECTS}

This paper presents a methodology to perform the visual quality control of cereal seeds samples. This operation called visual grading can be treated in three steps: acquisition, segmentation and classification. An acquisition system has been created to collect the data. Two new segmentation approaches have been developed. The proposed approaches have the same first step: a shape learning performed on isolated seed. The segmentation and classification methods are able to work with isolated seeds, low or high density of touching grain kernels.

Numerical results illustrate the interest of using superellipse approximation instead of ellipse. With a same sample in different disposition, the methods are able to find the same shape statistics. The results with the deterministic and probabilistic approaches have similar results in terms of quality measurements but the visual results present some differences. Further investigations are under way. Some defaults on the approaches happen in the cases of overlapping and of broken kernels. The first defaults can be solved using a confidence rate on the segmentation. The second defaults could be solved with additional learnings on broken grain kernels.

A comparative study with other approaches is under way. In the cereal industry, instead of visual grading, our approach could also be used for counting, identification, segmentation of a sample with different grain kernel species. The visual grading methodology can be applied to other objects than the cereals. Integration of more shape criteria to obtain better classifications is under consideration. Other acquisition modalities are also investigated: infrared and ultraviolet to detect information outside the visible range, stereovision and shape from shading to acquire 3D knowledge. 

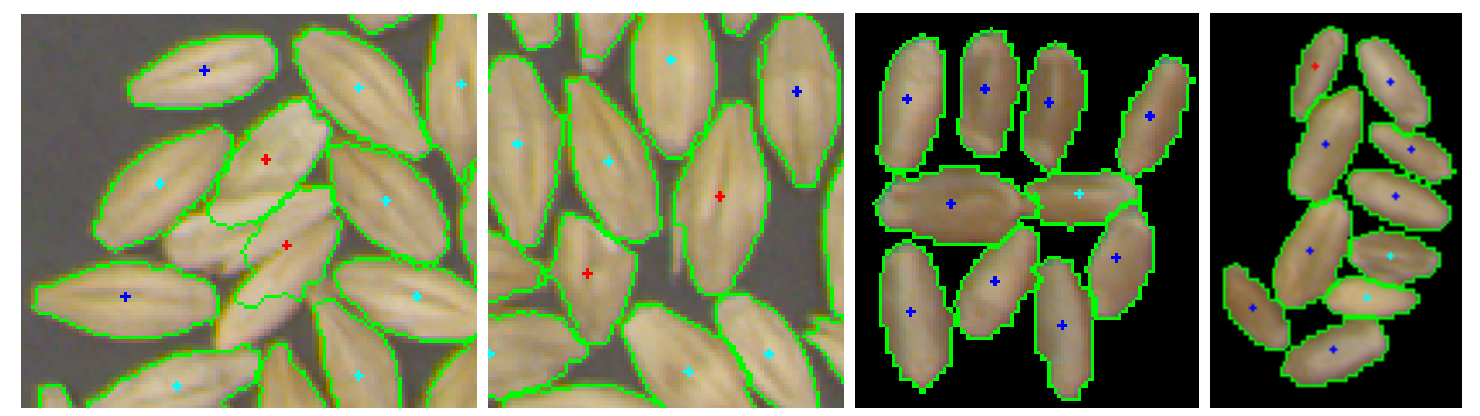

Figure 9. The blue, cyan and red crosses represent respectively the very good, good and bad confidences.

\section{ACKNOWLEDGEMENT}

This CIFRE thesis work was made possible thanks to the involvement of Alpha MOS company. We thank the members of the company who help us with the image acquisitions and provide us feedbacks on the software.

\section{REFERENCES}

[1] Kaur, H. and Singh, B., "Classification and grading rice using multi-class svm," International Journal of Scientific and Research Publications (IJSRP) 3, 1-5 (2013).

[2] Agustin, O. and Oh, B.-J., "Automatic milled rice quality analysis," in [Future Generation Communication and Networking], 2, 112-115 (2008).

[3] Yao, Q., Zhou, Y., and Wang, J., "An automatic segmentation algorithm for touching rice grains images," in [International Conference on Audio Language and Image Processing (ICALIP)], 802-805 (November 2010).

[4] Faessel, M. and Courtois, F., "Touching grain kernels separation by gap-filling," Image Analysis and Stereology 28(3), 195-203 (2011).

[5] Mebatsion, H. and Paliwal, J., "A fourier analysis based algorithm to separate touching kernels in digital images," Biosystems Engineering 108(1), 66-74 (2011).

[6] Zhang, G., Jayas, D. S., and White, N. D., "Separation of touching grain kernels in an image by ellipse fitting algorithm," Biosystems Engineering 92(2), 135-142 (2005).

[7] Gardiner, M., "The superellipse: a curve that lies between the ellipse and the rectangle," Scientific American 213(3), 222-232 (1965).

[8] Dubosclard, P., Larnier, S., Konik, H., Herbulot, A., and Devy, M., "Automatic method for visual grading of seed food products," in [International Conference on Image Analysis and Recognition (ICIAR)], Lecture Notes in Computer Science 1, 485-495 (2014).

[9] Dubosclard, P., Larnier, S., Konik, H., Herbulot, A., and Devy, M., "Deterministic method for automatic visual grading of seed food products," in [International Conference on Pattern Recognition Applications and Methods], (2015 (accepted, to appear)).

[10] Larnier, S., "Structure tensor based analysis of touching grain kernels for visual grading," Tech. Rep. 15019, LAAS (2015).

[11] Otsu, N., "A threshold selection method from gray-level histograms," IEEE Transactions on Systems, Man and Cybernetics 9, 62-66 (Jan. 1979).

[12] Hu, M., "Visual pattern recognition by moment invariants," Information Theory, IRE Transactions on $\mathbf{8}$, 179-187 (February 1962).

[13] Kuhl, F. P. and Giardina, C. R., "Elliptic fourier features of a closed contour," Computer graphics and image processing 18(3), 236-258 (1982).

[14] Bresson, X., Vandergheynst, P., and Thiran, J.-P., "A variational model for object segmentation using boundary information and shape prior driven by the mumford-shah functional," International Journal of Computer Vision 68(2), 145-162 (2006).

[15] Dice, L. R., "Measures of the amount of ecologic association between species," Ecology 26(3), 297-302 (1945). 